\title{
Classification of Motorcyclists without Helmet via Daubechies Wavelet Transform and SVM

\author{
${ }^{*}$ Sneha Ghonge, ${ }^{2}$ Jignyasa Sanghavi. \\ ${ }^{1,2}$ Department of Computer Science and Engineering, Shri Ramdeobaba College of Engineering and Management, \\ Nagpur, India \\ *Email: ghongesa@rknec.edu
}

\section{Received: 09 ${ }^{\text {th }}$ July 2018, Accepted: $14^{\text {th }}$ August 2018, Published: 31 ${ }^{\text {st }}$ August 2018}

\begin{abstract}
Nowadays, motorcycle accidents is on high rise and the main cause is traumatic brain injuries. Which suffers because most of the motorcyclists do not use helmet. In this paper we proposed a frame work for automatic detection of motorcyclists without helmet on public roads using surveillance videos. The frame work uses (SVM) support vector machine trained on features derived from the upper $60 \%$ part of the motorcyclists using Daubechies 8 wavelet transform. Then upper $60 \%$ part of the motorcyclists is isolated and then classified as helmet versus non - helmet using trained classifier. The performance of the proposed approach was evaluated on static photographs and videos. Experiment on real videos successfully detect 95.23\% of traffic rule violators and $97.33 \%$ on static photographs.
\end{abstract}

Keywords: Helmet Detection, Daubechies 8 Wavelet Transform, Saliency Map, (SVM) Support Vector Machine.

\section{Introduction}

In India, over $78 \%$ of vehicles on the road are twowheelers and they account for about $31.5 \%$ of road accident deaths, a statistic that has risen steeply over the years from $24.3 \%$ in 2014 to $26.8 \%$ in 2015 and $28.8 \%$ in 2016.Except two wheelers other road accidents are those in cars, taxis, van and other light medium motor vehicle (17.2\%), trucks (11.4\%), pedestrians (9.3\%) etc [1]. Dr. Mc Misra, director and dean of AIIMS says "Wearing a helmet and tying it properly can prevent loss of lives by 90 percent in motorcycle accident cases. We call it helmet vaccine," [2]. Helmet is safety armor for head, just like skull protects the brain from impacts helmet protects the head. The three magical layers of helmet thermoplastic layer, expanded polystyrene (eps) and inner soft spongy layer protect user from traumatic brain injuries [3]. National highway of traffic safety administration report says "The ratio of two-wheelers on public roads is $50 \%$ to that of human population". According to section 129 of the motorcycle vehicle act Government has made it mandatory to ride a motorcycle with helmet [4]. Nowadays traffic cops are capturing the photographs of the people not wearing helmet and necessary actions are taken against the violators but in this scenario human preference get the first priority .So automation of this process is on high requirement. And in the current scenario there are CCTV surveillance in every country so, implementation will also be cost effective.

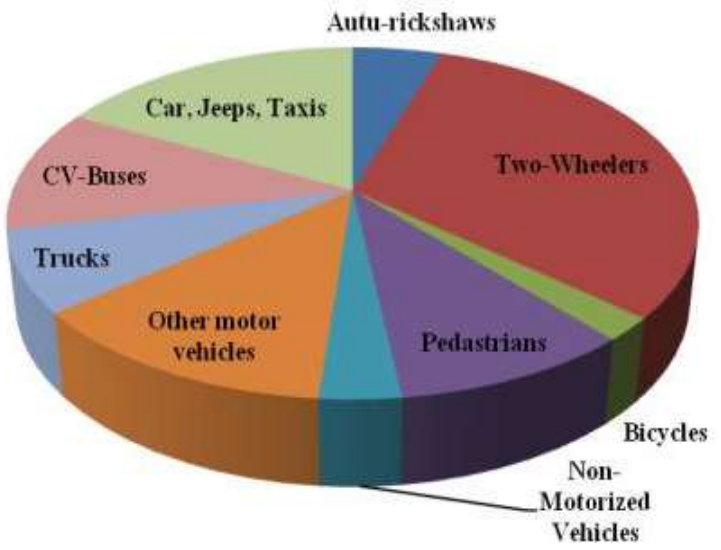

Fig 1: Total Number of Persons Killed in Road Accidents in India as per User Categories in the Year 2017

In this paper we present a frame work for detection of bike riders without helmet using (SVM) Support vector machine trained on features derived from the upper $60 \%$ part of the motorcycle and classifying using trained classifier. The remainder of the paper is organized as follows:

Section II reviews the related work for with their merits and demerits. Section III describes the proposed approach for automatic detection of motorcyclists with and without helmet. Section IV gives the information about dataset used, Experimental result and their analysis. At last the conclusion in section V.

\section{Literature Review}

Over the past years several researchers have been worked on traffic monitoring, including vehicle detection and classification and helmet detection in surveillance videos in real time. Wen et al. [5] proposed a system for detection of helmet using circular Hough transform. Presence of circular object 
in the scene classifies as helmet. As head is also circular sometimes it was mistaken between head and helmet. They applied this method to detect helmet in the surveillance system of automatic teller machine. Chiverton et al. [6] used edge information of the head region of the motorcycle using histogram of oriented gradients which will fed as in input to the classifier to classify whether the person is wearing helmet or not . Chiu et al. [7] used Canny edge detector[8]. Quantities of the edge points similar to the circle were classified as helmet. In [9] Silva et al. proposed a system in which it has used Kalman filter [10] to track the vehicle .The advantage of using Kalman filter was its ability to track motorcycle even if it is lightly occluded by another motorcycle. But Kalman filter fails because it works on linear static transition and to track objects we need non-linear static transition. Dahiya et al.[11] used two classifier one motorcycle and another for helmet. They used hand crafted features such as (HOG) Histogram of oriented gradients, (SIFT) Scale invariant feature transform, (LBP) Local binary pattern along with SVM $[12,13]$ for both classifiers. Their approach were promising but it failed to detect in certain environmental conditions. Doughmala et al. [14] proposed a system for detection of half and full helmet on the basis of Haar like features such as ear, mouth, nose, eyes, etc. Circular Hough transform was used for detecting the presence of helmet, but in this paper it has worked on fixed resolution images. The proposed approach is designed in such a way to overcome the above discussed limitations and suitable for real time application.

\section{Proposed Framework}

This section presents the proposed approach for real time detection of motorcyclists with and without helmet using both surveillance videos and static images. For that first the input the road video / images then using saliency map we segment our object of interest that is the motorcycle. After motorcycle has been extract the upper $60 \%$ part of the segmented image with an assumption that helmet lies mostly in that region. Next we extract the features of that upper extracted part using Daubechies 8 wavelet transform and these feature vector is then fed it as an input to the classifier to classify between the presence of helmet or not . Here we have used SVM classifier with linear kernel to train and evaluate our system. Fig.1 block diagram illustrates our proposed approach for detecting motorcyclists with and without helmet.

The steps of framework of proposed system and detailed description of each is as follows:

A. Object of interest segmentation using saliency map

B. Extract upper $60 \%$ of the segmented image.

C. Extract features using Daubechies 8 wavelet transform

D. Train SVM to classify between helmet and nonhelmet

(A) Object of interest segmentation using saliency map

Saliency Calculation

Saliency is determined by local contrast of image pixels with respect to its neighboring pixels at various scales. This is evaluated by the distance between the average feature vector of the pixels of sub-region of an image with that of neighboring pixels. Which gives a combined feature map ata given scale by using feature vectors for each pixel

[16]. The contrast based saliency value $C(i, j)$ of the image pixel is determined by the distance between the average vector of pixel feature of region R1 to that of region $\mathrm{R} 2$.

$$
C i, j=D\left[\left(\frac{1}{N 1} \sum_{p=1}^{N 1} V p\right)\right] \cdot\left[\left(\frac{1}{N 2} \sum_{q=1}^{N 2} V q\right)\right]
$$

Where $\mathrm{N} 1$ and $\mathrm{N} 2$ are the number of pixels in R1 and $\mathrm{R} 2$ respectively, $\mathrm{V}$ is vector corresponding to pixel and $\mathrm{D}$ is the Euclidean distance given by,

$\mathrm{D}=\|\mathrm{V} 1-\mathrm{V} 2\|$

Where $\mathrm{V} 1=[\mathrm{L} 1, \mathrm{a} 1, \mathrm{~b} 1] \mathrm{T}$ and $\mathrm{V} 2=[\mathrm{L} 2, \mathrm{a} 2, \mathrm{~b} 2] \mathrm{T}$ average vectors of region R1 and R2 . For an image of width $\mathrm{W}$ pixel and height $\mathrm{h}$ pixel the width of region $\mathrm{R} 2$ varies between:

$$
\frac{W}{2} \geq(W R 2) \geq \frac{W}{8}
$$

The final saliency map is calculated as the sum of salient values across scale $\mathrm{S}$,

$$
M i, j=\sum_{S} C i, j
$$

(B) Extract upper $60 \%$ of the segmented image For detecting the presence of helmet we are considering the upper $60 \%$ part of the motorcycle assuming that head of the motorcycle is present probably in this region we will extract the features of this upper part.

(C) Extract features of that upper extracted image using Daubechies 8 wavelet transform

Wavelet transform is an extension of Fourier transform rather the fact that Fourier transform works on single scale (time or frequency) and Wavelet transform works on multi- scale and also addresses the problem of non - stationary signals. Wavelet transform is a mathematical tool for decomposing a signal into time and frequency in a set of orthogonal waveform 
and it has two functions wavelet function and scaling function. To decompose the signal in different scale Discrete wavelet transform (DWT) has two filters low pass filter (LPF) and high pass filter (HPF)[17] . The output coefficient of a low pass filter are called as approximate and that of high pass filter are called as detailed [18]. There are various families of wavelet we have used daubechies ( $\mathrm{db} 8)$ wavelet due to its highest accuracy as compared to others.
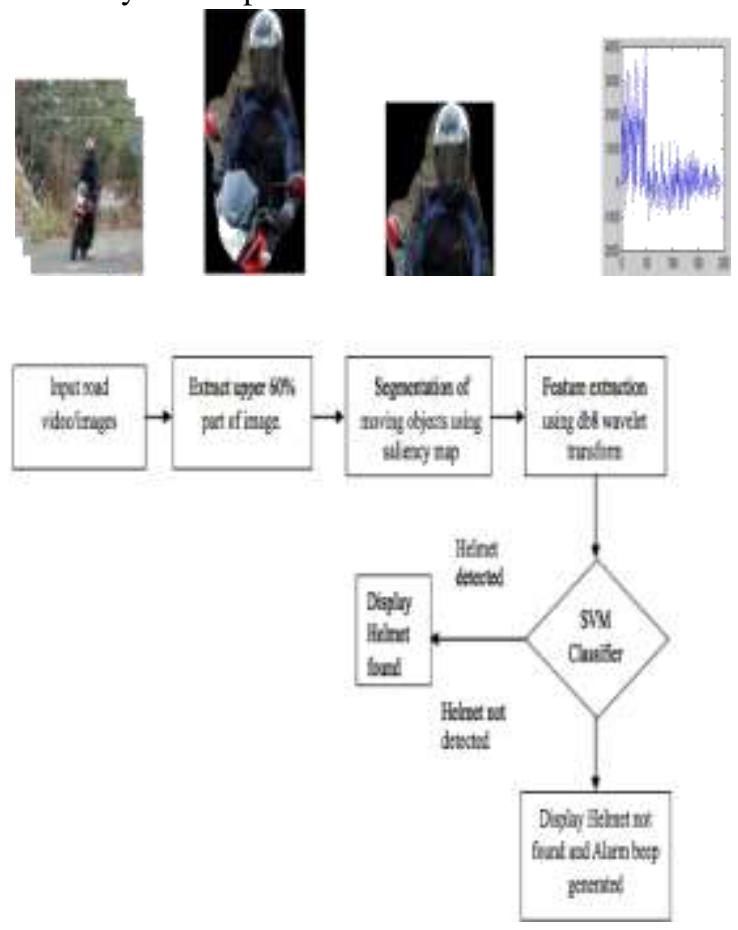

Figure 2: Flow Diagram of Framework for Detection of Motorcyclists with and without Helmet.

The equation of wavelet transform,

$$
\varphi a, b(t)=\frac{1}{\sqrt{a}} \varphi\left(\frac{t-b}{a}\right)
$$

the mother wavelet $\Psi$

(t) generates the other wavelet $\varphi a, b(t), a>0, b \varepsilon R$ of the family by change of scale a means by dilation and change of position $b$ means by translation .

Here we are using 196 features of an image/video. The main purpose was to obtain small feature vector and a good classification rate. For that 5 level daubechies 8 wavelet decomposition was performed. Original image of size $512 \times 512$ which was first decomposed into $256 \times 256$ in second level it decomposes to $128 \times 128$ in the third level to $64 \times 64$ and in the fourth level decomposition to $32 \times 32$ and finally in the fifth level to $16 \times 16$ features with an approximation we get 196 features in the last level that is the fifth level.

\section{(D) Train SVM to classify between helmet and non-helmet}

After feature extraction using wavelet transform now we classify between motorcycle versus nonmotorcycle by training the SVM based on the features derived using db8 wavelet transform. These feature vector will be fed as an input to the classifier which will then classify between helmet and non-helmet. The classifier used is SVM with linear kernel. We choose SVM due to its robustness and efficient classification performance over less number of trained data.

\section{Dataset and Experimental Results}

Dataset

We have evaluated our experiment on images as well as on videos for better efficiency. As the dataset was not available publically we captured our own images and videos. The dataset for images were captured by static camera at a location were number of motorcyclists with and without helmet were balanced .Total we capture 150 motorcyclists images. This images were captured at different angles to test the efficiency .For video data set we capture it with a static camera kept at a certain height from the road we collected 1 hour surveillance video for our testing purpose.

\section{Experiments}

Experiment was conducted on both videos and images. Videos: We have taken 1 hour surveillance video for our experiment. We kept first half of the video for training and the other half for testing whether it correctly classifies between helmet and non- helmet and a satisfactory results were obtained.

Images: Experiment was conducted on images to test the efficiency of images taken from different angles of the motorcyclists. Out of the 150 captured images we trained 90 images and the rest images were evaluated. Result shows that our proposed approach was correctly classified between helmets and non- helmet. All the program used for training and evaluating the images and videos data set of motorcyclists with and without helmet were programmed in MATLAB tool.

\section{Result}

In this section we present our experimental result and suitability of our proposed approach over the previously used approach .Below table show the experimental result performed on images and videos.

\begin{tabular}{|l|l|l|l|l|}
\hline Dataset & $\begin{array}{l}\text { Proposed } \\
\text { method }\end{array}$ & $\begin{array}{l}\text { Total Images } \\
\text { /Videos }\end{array}$ & $\begin{array}{l}\text { Correctly } \\
\text { tested }\end{array}$ & Efficiency \\
\hline Images & $\begin{array}{l}\text { Db8 } \\
\text { SVM }\end{array}$ & 150 & 146 & $97.33 \%$ \\
\hline Videos & $\begin{array}{l}\text { Db8 } \\
\text { SVM }\end{array}$ & $\begin{array}{l}1 \text { hour (42 } \\
\text { motorcyclists) }\end{array}$ & 40 & $95.23 \%$ \\
\hline
\end{tabular}

Table 1: Performance of Classification of Motorcyclists with helmet and without Helmet. 


\section{Conclusion}

In this paper we proposed an approach for automatic detection of bike riders with and without helmet which will help the traffic police for detecting such violators and take necessary action against them. In the proposed has met certain challenges related to poor quality of the videos and environmental conditions. One future work can be the automatic detection of these violators and if somebody is found violating the rules then trace the license number plate of the vehicle and automatic actions to be taken. No manual work should be done which will in turn increase the efficiency and time awareness among the violators.

\section{References}

[1] Maya Sharma, "use your head - use a helmet", Team NDTV article ,Dec 12, 2016.

[2] Posted on motor vehicle act " why is it so important to wear helmet on motorcycles?", mainor wirth injury lawyers, http:// mainworth.com , Nov $23,2017$.

[3] TakshakDawda, NDTV Article " Twowheelers are commercial vehicles see highest road accidents fatalities" , July 10, 2017.

[4] Anisha Bhatia ," Wearing helmets - A choice between life and death" Team NDTV article , Dec 9, 2016.

[5] C. Y.Wen, S.H.Chiu, J.J.Liaw, and C.P. Lu, "The safety helmet detection for ATM's surveillance system via the modified hough transform" in IEEE 37th Annual International Carnahan Conference on Security Technology , 2003 , pp- 364-369.

[6] J. Chiverton , "Helmet presence classification with motorcycle detection and tracking," IET Intelligence Transport Systems (ITS), Volume 6 , no. 3 , pp. 259-269, 2012.

[7] C.C. Chiu, M.Y. Ku, and H. -T. Chen, "Motorcycle detection and tracking system with occlusion segmentation," in Proceeding of International Workshop on Image Analysis for Multimedia Interactive Services , Santorini , Greece, 6-8 June 2007, pp. 32-32.

[8] J. Canny, "Finding edges and lines in images," Cambridge, MA, USA,Tech. Rep., 1983.

[9] R. V. Silva , T. Aires , and V. Rodrigo ," Helmet Detection on Motorcyclists using image descriptors and classifiers", in Proceeding of Graphics , Patterns an Images ( SIBGRAPI), Rio de Janeiro ,Brazil , 27-30 August 2014 , pp. 141-148.

[10] R. E. Kalman, "A new approach to linear filtering and prediction problems," Journal of Basic Engineering, vol.82, no. 1, pp. 3545,1960.
[11] K. Dahiya, D. Singh and C.K .Mohan, "Automatic detection of bike riders without helmet using surveillance videos in real -

time", in Proceeding of International Joint Conference Neural Networks (IJCNN), Vancouver, Canada , 24-2 July 2016,pp.30463051.

[12]C. Cortes and V. Vapnik, "Support vector networks," Machine Learning(Springer), vol. 20, no. pp.273-297, 1993.

[13] D. Singh, D. Roy, and C. K. Mohan, "Dip-svm: distribution preserving kernel support vector machine for bigdata,'IEEE Trans. on BigData,2017.[Online].Available:http://dx.doi. org/10.1109/TBDATA.2016.2646700.

[14] Pathasu Doughmala, Katanyoo Klubsuwan, "Half and Full Helmet Detection in Thailand using Haar Like Feature and Circle Hough Transform on Image Processing" in Proceeding of IEEE International Conference on Computer and Information Technology, Thailand, Bangkok ,pg. 611-614,2016.

[15] Radhakrishna Achanta, Francisco Estrada," Salient Region Detection and Segmentation" International journal of crime and victim survey " 2008.

[16]Chenlei Guo, Qi Ma," Spatio-temporal Saliency Detection Using Phase Spectrum of Quaternion Fourier Transform, IEEE , 2008.

[17] Apatean (discant), a. Rogozan, a. Bensrhair,"Wavelets as features for objects recognition" Proceedings of the IEEE international Conference on automation, quality and testing, robotics, Theta 16, cluj-napoca, romania, 2008.

[18]M.P. Nageswari , U. Sabura banu ," Feature extraction of ecg using daubechies wavelet and classification based on fuzzy c-means clustering technique", proceeding of national conference on control, communication and information technology, 6 may 2013. 